\title{
Improving WLAN Performance by Modifying an IEEE 802.11 Protocol
}

\author{
Nurul I. Sarkar, Auckland University of Technology, New Zealand
}

\begin{abstract}
One of the limitations of the IEEE 802.11 distributed coordination function (DCF) protocol is its low bandwidth utilization under medium-to-high traffic loads resulting in low throughput and high packet delay. To overcome performance problems, traditional IEEE 802.11 DCF ("DCF") protocol is modified to the buffer unit multiple access (BUMA) protocol. The BUMA protocol achieves a better system performance by introducing a temporary buffer unit at the medium access control (MAC) layer to accumulate multiple packets and combine them into a single packet (with a header and a trailer) before transmission. This paper provides an in-depth performance evaluation (by simulation) of BUMA for multiuser ad hoc and infrastructure networks. Results obtained show that the BUMA is more efficient than that of DCF. The BUMA protocol is simple and its algorithm (software) can be upgraded to 802.11 networks requiring no hardware changes. The BUMA protocol is described and simulation results are presented to verify the performance.
\end{abstract}

Keywords: $\quad$ BUMA Protocol, Distributed Coordination Function (DCF), IEEE 802.11, Medium Access Control (MAC) Protocol, User-Datagram Protocol (UDP)

\section{INTRODUCTION}

IEEE 802.11 based wireless local area networks (WLANs) are gaining widespread popularity due to their simplicity in operation, robustness, low cost, well-defined standard (e.g. $802.11 \mathrm{~b} / \mathrm{g}$ ) and user mobility offered by the technology.

A good wireless MAC protocol should provide an efficient mechanism for sharing a limited wireless channel bandwidth, together with simplicity of operation, high bandwidth utilization and network fairness (i.e. equality in channel access). Ideally low mean packet delay,

DOI: 10.4018/ijwnbt.2011010102 high throughput, low packet drop ratio, and a good fairness under high-traffic loads are desired, but in reality the 802.11-based WLANs do not provide all the quality of service (QoS) provisions simultaneously. Therefore, various MAC protocols have been developed to suit different applications where various tradeoff factors have been considered (Aad, Ni, Barakat, \& Turletti, 2005; Chieochan, Hossain, \& Diamond, 2010; Li \& Chen, 2010; Luo, Rosenberg, \& Girard, 2010). A study of the performance of 802.11 under high-traffic loads is required to assist efficient MAC protocol design for WLANs to achieve a better QoS in terms of high throughput and low packet delay in such systems. 
The 802.11 standard defines two types of MAC schemes: Distributed Coordination Function (DCF) and Point Coordination Function (PCF). DCF is defined as a mandatory MAC protocol while PCF is optional. In this paper we focus on the DCF mode in 802.11 which has been widely deployed because of its simplicity. In the DCF, wireless stations (STAs) communicate with each other using a contention-based channel access method known as Carrier Sense Multiple Access with Collision Avoidance (CSMA/CA).

The performance of the DCF has been analyzed in numerous papers (Banchs, Serrano, \& Azcorra, 2006; Bianchi, 2000; David, Ken, \& Doug, 2007; Kuo, 2007). Clearly, the DCF has several limitations. Primarily, it does not perform well under medium-to-high traffic loads. If the number of active users increases, the mean packet delay, throughput, fairness and packet drop ratio of the 802.11 degrade significantly.

The service differentiation and fairness issues of 802.11 have been discussed in (Banchs \& Vollero, 2006; Ferre, Doufexi, Nix, \& Bull, 2004; Lin \& Wu, 2007; Sarkar, 2006). The 802.11e task group is proceeding to build the QoS enhancements of the 802.11 (IEEE Standards Association, 2005). Although various innovative MAC protocols have been developed and reported in the computer networking literature, very few protocols satisfy simultaneously all the QoS provisions while retaining simplicity of implementation in real WLANs. This paper proposes an enhancement of DCF called buffer unit multiple access (BUMA) protocol to overcome the limitations of DCF mentioned above. The BUMAprotocol is developed through minor modifications of the existing DCF.

The proposed BUMA protocol provides higher throughput, lower packet delay, lower packet dropping, and greater fairness under medium-to high loads than that of DCF. The better system performance is achieved by introducing a temporary buffer unit at the MAC layer for each active connection on the network, accumulating multiple packets (for example, three packets) and combining them into one large packet with a single header for transmissions. The BUMA can be used to improve the performance of 802.11 networks, including the $802.11 \mathrm{~b} / \mathrm{g}$, and would be a good candidate for providing real-time multimedia services.

The remainder of this paper is organized as follows. We first provide an overview of DCF protocols. We then summarize past research on enhancements of the original 802.11 networks. The proposed BUMA protocol is then described. The simulation environment and parameter settings are presented. The performance of the BUMA is then compared with that of the DCF. Finally, the system implication is discussed and a brief conclusion concludes the paper.

\section{THE BASIC DCF PROTOCOL}

The basic operation of the DCF is illustrated in Figure 1. In the basic access mode, a station monitors the channel until an idle period equal to a DCF inter-frame space (DIFS) is detected before transmission of a frame. If the channel is found to be busy, the station defers (and continues listening to the channel) until the channel becomes idle for at least a DIFS. Then the station begins its backoff time to avoid collisions. After a successful backoff time the station transmits a packet.

The collision avoidance technique adopted in the DCF is based on a binary exponential backoff (BEB) method, which is implemented in each station by means of a parameter known as the backoff counter. The backoff time is used to initialize the backoff counter. This counter is decremented only when the medium is idle and is frozen when activity is sensed. The backoff counter is periodically decremented by one time slot each time the medium sensed is idle for a period longer than a DIFS. A station transmits a packet when its backoff counter is zero. The random backoff time can be computed using (1) (IEEE Standards Association, 1999):

Backoff time (BT) = Random () X aSlotTime

Copyright (C) 2011, IGI Global. Copying or distributing in print or electronic forms without written permission of IGI Global is prohibited. 
Figure 1. Basic access method of DCF protocol

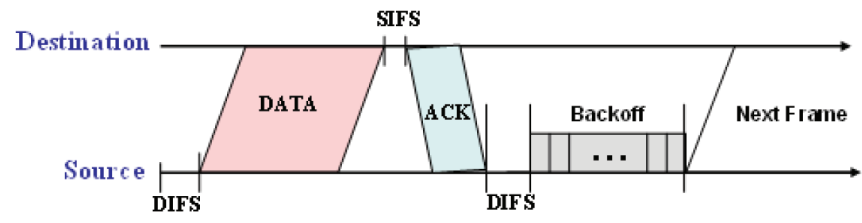

Where, Random () is an integer randomly chosen from a uniform distribution over the interval $[0, \mathrm{CW}-1], \mathrm{CW}$ is the contention window size.

At the first transmission attempt $\mathrm{CW}=$ CWmin, and it is doubled at each retransmission up to CWmax. In the IEEE 802.11 DSSS (direct sequence spread spectrum) specification, $\mathrm{CWmin}=31$ and $\mathrm{CWmax}=1023$. More details about backoff algorithm can be found in (IEEE Standards Association, 1999a; Yun, Ke-Ping, Wei-Liang, \& Qian-Bin, 2006).

\section{SHORTCOMINGS OF DCF PROTOCOL}

Although DCF has been standardized and is gaining widespread popularity as a channel access protocol for WLANs, the protocol has several potential limitations. One of the limitations of the DCF protocol is the low bandwidth utilization under the medium-to-high loads, and consequently, it achieves low throughput, high packet delay and high packet drop ratios. Another deficiency of the DCF protocol is poor fairness in sharing a channel bandwidth among the active stations on the network. High transmission overhead (headers, inter-frame spaces, backoff time and acknowledgments) is also a fundamental problem in the DCF protocols (Xiao, 2004). The proposed BUMA protocol overcomes these shortcomings of DCF.

\section{PRIVIOUS WORK ON THE ENHANCEMENT OF DCF}

This section summarizes previous work on improvements to the original DCF protocol. Many network researchers have proposed methods to improve the protocol's performance. In this paper, for brevity, we refer to only a selected set of literature that is indicative of the range of approaches used to improve throughput, packet delay, and fairness performance.

Bharghavan et al. (1994) proposed a MAC protocol called multiple access with collision avoidance for wireless (MACAW) in alleviating the fairness problem of DCF. This fairness improvement is mainly due to the selection of a better backoff algorithm, called multiplicative increase and linear decrease (MILD), and some additional control packets in the system.

Xiao (2004) proposed two mechanisms, namely, concatenation and piggybacking in order to reduce the overhead of the DCF protocols. The idea is to concatenate multiple frames in a station's queue before transmission into the medium. Under the piggyback scheme, a receiving station piggybacks a data frame to a transmitting station as long as the receiver has a frame for transmission.

Cali and Conti (2000) proposed an improvement to the DCF protocol called dynamic 802.11, which is a distributed algorithm for altering backoff window size. By observing the status of the channel, a station obtains an estimate of the network traffic and uses this estimate to tune the backoff window sizes.

Bianchi, Borgonovo, Fratta, Musumeci, and Zorzi (1997) developed a MAC protocol for WLANs, C-PRMA. An algorithm for adaptive contention window size is proposed for DCF (Bianchi, 2000; Bianchi, Fratta, \& Oliveri, 1996). The key concept is to dynamically select the optimum backoff window size based on an estimate of the number of contending stations. This optimization is performed through the 
measurement of channel activity by each station. They showed that by using this adaptive contention window size, instead of BEB, the DCF could be significantly improved, especially at high loads and large user numbers.

Bruno and Conti (2002) proposed an enhancement of the DCF called p-persistent 802.11. The improvement is mainly due to the selection of a better algorithm for selecting the backoff interval. Instead of the BEB used in the original DCF, the backoff interval of the p-persistent 802.11 is sampled from a geometric distribution with parameter $p$.

Natkaniec and Pach (2002) developed the MAC protocol called Priority Unavoidable Multiple Access (PUMA) to improve the performance of DCF. The key concept is to introduce a priority scheme for time bounded services. Both a special jam signal and an additional timer are required to support isochronous transmission. Network performance is improved using a new backoff algorithm called double increment double decrement (DIDD) (Natkaniec \& Pach, 2000).

You et al. (2003a, 2003b) proposed two MAC methods for improving DCF, namely carrier sense multiple access with ID countdown (CSMA/IC) (You et al., 2003a) and carrier sense multiple access with collision prevention (CSMA/CP) (You et al., 2003b). In CSMA/IC, the transmission radius is fixed to a certain unified value and the sensing radius is twice or more than the transmission radius. By proper station synchronization, only two stations can compete for a medium at a time and the station with a packet that has a larger unique ID has priority. By exchanging synchronizing packets, CSMA/ IC can be $100 \%$ collision free even in random access environments. In CSMA/CP, the wireless channel is partitioned into a control channel and several data channels. The key concept in achieving $100 \%$ collision free transmission is to prevent collisions in the control channel.

Kwon, Fang, and Latchman (2003) developed an efficient MAC algorithm called the fast collision resolution (FCR) to resolve the collisions by increasing the size of the contention window for both the colliding and deferring stations. All active stations redistribute their backoff timers to avoid possible future collisions.

Jagadeesan, Manoj, and Murthy (2003) proposed a MAC protocol suitable for wireless ad hoc networks known as Interleaved Carrier Sense MultipleAccess (ICSMA). This protocol uses two data channels (channel 1 and 2) of equal bandwidth, and the handshaking process is distributed between the two channels. A station is allowed to transmit using either channel 1 or 2. For example, a source station may send an RTS packet to a destination station over channel 1, and the destination station responds with CTS on channel 2. ICSMA uses the same binary exponential backoff mechanism as DCF, including waiting times such as DIFS, and PIFS. The ICSMA performs better than the DCF protocol with exposed stations with respect to throughput, packet delay, and fairness.

Ozugur, Naghshineh, Kermani, and Copeland (1999) proposed a $\mathrm{p}_{\mathrm{ij}}$-persistent CSMAbased backoff algorithm for load balancing among the wireless links, improving the fairness performance of DCF. Each station calculates a link access probability $\mathrm{p}_{\mathrm{ij}}$ using either a connection-based (i.e. the information on the number of connections it has with its neighboring stations) or a time-based method (based on the average contention period).

Jiang and Liew (2008) investigated methods of improving throughput and fairness of the DCF by reducing both the exposed and hidden station problems. They showed that the DCF network is not scalable due to exposed and hidden station problems, more access points (APs) do not yield higher total throughput. By removing these problems, it is possible to achieve a scalable throughput.

Wang and Garcia-Luna-Aceves (2003) proposed a hybrid channel access method to alleviate the fairness problem of DCF without sacrificing much throughput and simplicity. The protocol is based on both sender-initiated and receiver-initiated handshakes. 
The previous approaches on the enhancement of DCF protocols reviewed in this section are grouped into four main categories shown in Table 1.

\section{THE PROPOSED BUMA PROTOCOL}

The proposed BUMA protocol described in this section differs from the earlier work. BUMA is implemented through minor modifications of DCF (IEEE Standards Association, 1999b). The design of BUMA was motivated by the key idea that a typical WLAN can increase throughput by sending a payload using fewer but longer packets (Choudhury \& Gibson, 2007; Ergen \& Varaiya, 2008; Xiao, 2004). With fewer packets used to deliver the same payload, proportionally less time is spent in the backoff state. Many network researchers have highlighted this aspect of network performance improvement (Chatzimisios, Vitsas, \& Boucouvalas, 2002; Ergen \& Varaiya, 2008; Ganguly et al., 2006; Wang, Liew, \& Li, 2005).

In BUMA, for each active connection a temporary buffer unit is created at the MAC layer where multiple packets are accumulated and combined into a single packet (with a header and a trailer) before transmission. Assuming a realistic wireless Ethernet packet length of 1,500 bytes, the optimum length of the buffer unit was empirically determined to be that of three 1500-byte packets plus header and trailer. The optimization of buffer unit length is discussed later in this section.

The number of buffer units is determined by the number of active connections between the source and destination stations. Each link has its own buffer unit, and each buffer unit stores one or more packets where each packet appears as a MAC Protocol Data Unit (MPDU) in the MAC layer with the same destination address. Thus, the content of a buffer unit is a large packet that appears as a MAC Segment Data Unit (MSDU) in the MAC layer with a single header and a trailer. Now the question arises about the maximum length of the combined packet (i.e. length of an MSDU).

For both wired and wireless Ethernet LANs, the maximum length of a MAC frame is 2,346 bytes, which is a fragmentation threshold. The mean packet length is about 1,500 bytes with payload length ranges from 46 to 1,460 bytes. In the optimized BUMA (BUMA ${ }_{\text {opt }}$, the maximum length of a buffer unit is 4,534 bytes, accommodating three 1,500-byte packets plus a 34-byte envelope (MAC header and cyclic redundancy check (CRC)). In such cases, the MSDU would be fragmented into two frames before transmission since its length is greater than the fragmentation threshold.

When a station fills the buffer unit, it first schedules the packet and then puts the next set of packets in the empty buffer unit from the same link. Under medium-to-high traffic loads, each station will always have packets for

Table 1. Categories of MAC approaches reviewed

\begin{tabular}{|c|c|}
\hline Approach & Example of MAC proposals \\
\hline Packet concatenation & 802.11 DCF improvement (Xiao, 2004). \\
\hline $\begin{array}{c}\text { Optimization of contention } \\
\text { window size and/or backoff } \\
\text { algorithms. }\end{array}$ & $\begin{array}{c}\text { MACAW (Bharghavan, 1994), PUMA (Natkaniec \& Pach, 2002), FCR (Kwon } \\
\text { et al., 2003), p-persistent 802.11 (Bruno et al., 2002), dynamic 802.11 (Cali \& } \\
\text { Conti, 2000), C-PRMA (Bianchi et al., 1997), Pij-persistent backoff (Ozugur et } \\
\text { al., 1999). }\end{array}$ \\
\hline $\begin{array}{c}\text { Using a control channel for } \\
\text { handshaking and/or collision } \\
\text { prevention. }\end{array}$ & $\begin{array}{c}\text { CSMA/CP (You et al., 2003b), CSMA/IC (You et al., 2003a), ICSMA (Jaga- } \\
\text { deesan et al., 2003), Hybrid channel access (Y. Wang \& Garcia-Luna-Aceves, } \\
\text { 2003). }\end{array}$ \\
\hline $\begin{array}{c}\text { Solving hidden and exposed } \\
\text { station problems. }\end{array}$ & 802.11 fairness improvement (Jiang \& Liew, 2008). \\
\hline
\end{tabular}


transmission and the buffer unit will be filled up with packets quickly within a time interval. When traffic is low, BUMA (Figures 2 and 3) will perform as well as DCF by reducing the buffer unit length to one packet. DCF is effectively a special case of BUMA where the buffer unit length is one packet. Therefore, in the proposed method, the mean packet delay is bounded since a packet will not remain in the buffer permanently while waiting for the second and subsequent packets to arrive.

The basic operation and the frame structure of the BUMA protocol are illustrated in Figures 2 and 3, respectively. Buffer unit contains multiple MPDUs (Figure 3). The actual number of MPDUs in a buffer unit will depend on packet length supported by upper protocol layers. For instance, for the transmission of a 500-byte IP datagram, a maximum of nine
MPDUs would be stored in a buffer unit of 4,500 bytes.

The proposed buffer unit mechanism has several benefits. Firstly, it transmits a greater payload (by scheduling a larger packet) and consequently achieves better throughput than DCF. Secondly, by adopting the buffer unit mechanism one can achieve higher bandwidth utilization and better fairness than in DCF because it wastes less potential transmission time in the backoff and channel contention processes. Referring to the example of the 500byte IP datagram, instead of nine contention periods, only one contention period is needed to transmit nine IP datagrams. BUMA, therefore, dramatically reduces the average packet contention delay, especially for shorter packet lengths, while maintaining better throughput by transmitting a combined packet. Finally,

Figure 2. Basic operation of the BUMA protocol

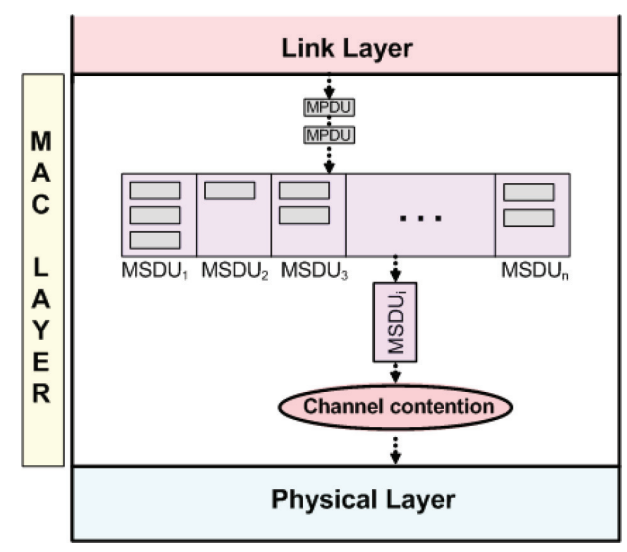

Figure 3. Frame structure of the BUMA protocol

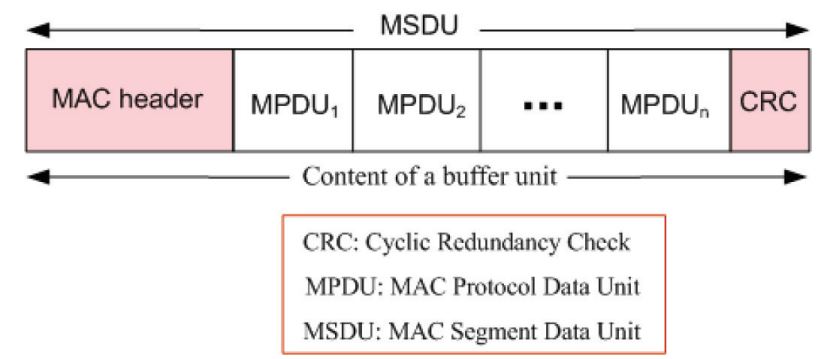

Copyright (C) 2011, IGI Global. Copying or distributing in print or electronic forms without written permission of IGI Global is prohibited. 
the packet transmission overhead will be reduced significantly. Without the buffer unit mechanism, each packet transmission requires a separate set of overheads, including headers, inter-frame spaces, backoff time, CRC and acknowledgements; in contrast, only one set of overheads would be used with the buffer unit mechanism. However, all these benefits come with a trade-off, a small processing delay at the stations. The transmission overheads and throughput analysis of BUMA are presented. The processing delay at the stations is also addressed in this section.

Although the BUMA protocol is based on the concepts of concatenation mechanism proposed in (Xiao, 2004), it differs in significant ways. The BUMA protocol is simple and does not require any additional control packets to deliver a combined packet (up to three packets), whereas (Xiao, 2004) requires three additional control frames (for example, a frame control type, concatenated frame count, and a total length field) to deliver a super-frame. In addition, we study the performance of BUMA protocol under high traffic loads, and have introduced two new metrics namely, packet drop ratio and fairness.

\section{OPTIMIZATION OF BUFFER UNIT}

Yin, Wang, and Agrawal (2005) investigated the optimal packet length that maximizes the DCF throughput under different channel conditions and traffic loads. A trade-off exists between a desire to reduce the overhead by adopting larger packet length, and the need to reduce packet error rates in the error-prone environments by using smaller packet length. For example, for an error-prone channel with a bit-error rate (BER) of $2 \times 10^{-5}$, the throughput reaches the maximal value of 0.6049 for a packet length of 900 bytes. However, the optimal packet length varies with the change of traffic load, and the optimal packet length under light loads is larger than the optimal packet length under high loads.
For an ideal channel condition, both throughput and packet delay increase with the packet length. Therefore, to achieve the best mean delay and throughput performance we have chosen a packet length of 1,500 bytes (a realistic figure close to the wired Ethernet). The mean throughput and packet delay of the BUMA protocol depends on the length of a buffer unit. Now the question arises as to the optimal buffer unit length required to achieve the best mean delay and throughput performance. We have determined the optimal length of the buffer unit empirically. We observe that the throughput performance of the BUMA protocol increases slightly with increasing buffer unit length and then saturates at buffer unit length of 10 or more packets. On the other hand, the lowest mean packet delay is achieved at a buffer unit length of three packets. By considering both the throughput and packet delays we have chosen three packets as the optimum buffer unit length.

\section{STRENGTHS AND WEAKNESSES OF THE BUMA}

The BUMA protocol provides better bandwidth utilization (Figure 4) than the DCF because it wastes less transmission capacity in the backoff state, and consequently, it achieves high throughput, low packet delay, low packet drop ratio, and good fairness especially under medium-to-high loads. In addition, the BUMA requires less overhead to send the same amount of payload than the DCF. This improvement is due to the BUMA protocol's scheduling strategy in which a single header and a trailer is required for scheduling multiple packets. Moreover, the BUMA protocol is simple and can easily be implemented in the DCF without changing any existing hardware.

Although the BUMA protocol provides a better fairness than the DCF, it does not provide $100 \%$ fairness in sharing a channel's bandwidth among the active stations on the network.

In the case of $\mathrm{BUMA}_{\text {nonopt }}$, the maximum allowable MSDU is set to the wireless Ethernet 
Figure 4. Throughput comparison of the $802.11 \mathrm{~b}$ DCF, BUMAnonopt and BUMAopt protocols for a single user network

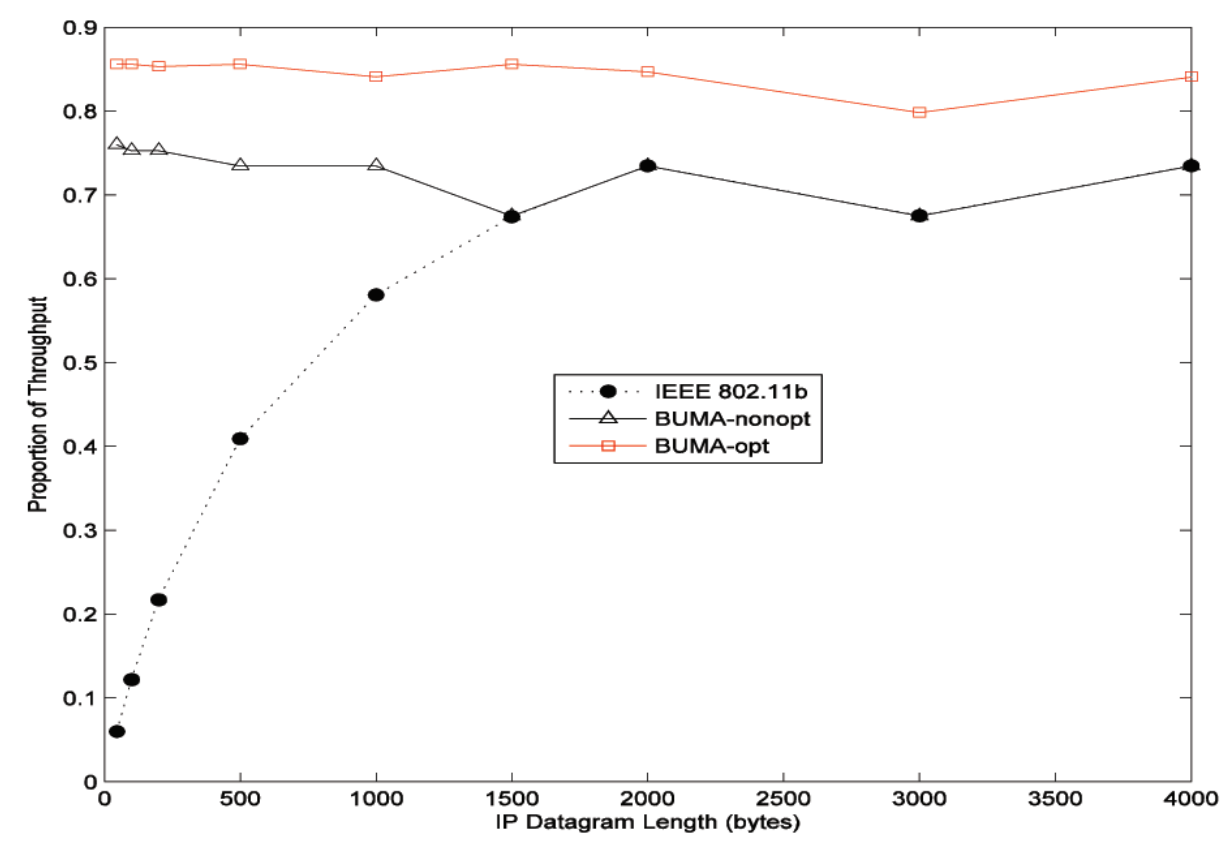

fragmentation threshold (so that frames can be transmitted without fragmentation). As shown in Figure 4, BUMA ${ }_{\text {nonopt }}$ achieves higher throughput than DCF for payload lengths $\leq 1,000$ bytes i.e. the throughput gain is more significant for short payloads (see above Example). Sending a larger payload with the same set of overheads causes this improvement. However, for payloads $>1,000$ bytes, BUMA $A_{\text {nonopt }}$ does not improve on DCF since the length of the Ethernet fragmentation threshold limits the performance.

Now in the case of BUMA ${ }_{\text {opt }}$, the optimum length of the buffer unit (4,534 bytes) becomes the maximum allowable MSDU. BUMA ${ }_{\text {opt }}$ offers higher throughput than DCF irrespective of payload length. This is a notable result that clearly demonstrates the superiority of both BUMA $_{\text {nonopt }}$ and BUMA $A_{\text {opt }}$ over DCF. Also, BUMA's throughput is almost independent of IP datagram length, unlike that of DCF.

By comparing BUMA $A_{\text {nonopt }}$ and BUMA ${ }_{\text {opt}}$, one can observe that BUMA ${ }_{\text {opt }}$ offers 10 to
$18 \%$ greater throughput than BUMA ${ }_{\text {nonopt }}$ for payload lengths smaller than 4,000 bytes. This throughput improvement is as a result of BU$\mathrm{MA}_{\text {opt }}$ transmits a slightly larger payload than BUMA $_{\text {nonopt }}$ with the same set of overheads.

\section{SIMULATION ENVIRONMENT AND PARAMETERS}

A simulation model was developed using ns-2 simulator (www.isi.edu/nsnam/ns) to study the performance of the proposed BUMA protocol. All stations communicate using identical half-duplex wireless radio based on the DCF, with data rate set at $11 \mathrm{Mbps}$. The RTS/CTS mechanism was turned off. Stations were stationary. The transmission and carriersensing ranges were set to $250 \mathrm{~m}$ and $550 \mathrm{~m}$, respectively. The ad hoc on-demand distance vector (AODV) routing protocol and the tworay ground propagation model were used. 
Streams of data packets generated at stations were modeled as independent Poisson processes with an aggregate mean packet generating rate $\lambda$ packets/s. All data sources are user-datagram protocol(UDP) traffic streams with fixed packet length of 1500 bytes. To simplify the simulation model, we consider a perfect radio propagation environment in which there is no transmission error due to interference and noise in the system, and no hidden and exposed station problems.

\section{Simulation Parameters}

Table 2 lists the parameter values used in the simulation of both the BUMA and 802.11 DCF protocols. Each simulation run lasted for 60 seconds simulated time in which the first 10 seconds was the transient period. The observations collected during the transient period are not included in the final simulation results.

\section{MODEL VALIDATION}

The ns-2 simulation results presented in this paper were verified in several ways. First, the simulation model was validated through radio propagation measurements from wireless laptops and APs for 802.11b (Sarkar \& Sowerby,
2006; Siringoringo \& Sarkar, 2009). A good match between simulation results and real measurements for $\mathrm{N}=2$ to 4 stations validates the simulation model. Second, ns-2 results were compared with the results obtained from OPNET Modeler (OPNET Modeler) and a good match between two sets of results further validated the simulation models.

\section{PERFORMANCE RESULTS AND COMPARISON}

We consider four important network performance metrics: (1) throughput; (2) packet delay; (3) fairness; and (4) packet drop ratio; for both individual stations and the overall network.

The throughput (measured in Mbps) is the mean rate of successful message delivery over a communication channel. The mean packet delay is defined as the average time (measured in seconds) from the moment the packet is generated until the packet is fully dispatched from that station. This packet delay includes queuing delay and medium access delay at the source station, and packet transmission time.

We define a metric for fairness measurement called mean deviation of throughput (MDT) as follows:

Table 2. Simulation parameters

\begin{tabular}{|c|c|}
\hline Parameter & Value \\
\hline Data rate & $11 \mathrm{Mbps}$ \\
\hline Basic Rate & $2 \mathrm{Mbps}$ \\
\hline Wireless cards & $802.11 \mathrm{~b}$ \\
\hline Slot duration & $20 \mu \mathrm{s}$ \\
\hline SIFS & $10 \mu \mathrm{s}$ \\
\hline DIFS & $50 \mu \mathrm{s}$ \\
\hline Simulation time & 60 seconds \\
\hline Packet/Traffic type & UDP \\
\hline Application & CBR \\
\hline PHY modulation & DSSS \\
\hline CWmin & 31 \\
\hline CWmax & 1023 \\
\hline
\end{tabular}




$$
M D T=\frac{\sum\left|\left(T_{i}-\bar{T}\right)\right|}{N}
$$

Where $\mathrm{T}_{i}$ is the throughput at station $i ; \bar{T}$ is the network wide mean throughput; and $\mathrm{N}$ is the number of active stations.

MDT is the spread or variation of individual stations' throughput from the network wide mean throughput. For instance, a MAC protocol is said to be $100 \%$ fair if MDT is zero (i.e. $T_{i}=\bar{T} \forall i$ ). The value of MDT indicates the level of unfairness of a MAC protocol. Hence a MAC protocol with a smaller MDT is desirable. MDT was used to compare the fairness of BUMA and DCF.

We define another metric called packet drop ratio $\left(P_{d r}\right)$ as follows.

$$
P_{d r}=\frac{N_{p d}}{N_{t p}}
$$

Where, $N_{p d}$ is the total number of packets dropped at the network, which is the difference of the total transmitted packets and successfully received packets; and $N_{t p}$ is the number of transmitted packets at the destination stations.

The $P_{d r}$ is directly related to packet collision rates (i.e. high packet collisions at the destination stations result in higher packet drop ratio). This metric tells us about the capacity of a MAC protocol in delivering packets successfully to the destination stations. A MAC protocol with a low packet drop ratio is desirable.

We present the empirical results obtained from simulation runs for both the proposed BUMA and the DCF. We demonstrate the performance of the BUMA protocol by considering both ad hoc and infrastructure networks with UDP traffic operating under uniform loads. The simulation results report the steady-state behavior of the network and have been obtained with the relative error $<1 \%$, at the $99 \%$ confidence level.

\section{Throughput Performance}

The network throughput versus offered load performance of BUMA and DCF with $\mathrm{N}=40$ stations for an ad hoc and infrastructure network is shown in Figure 5 (a) and (b), respectively. One can observe that BUMA provides higher throughput than DCF irrespective of network architecture, especially under medium-to-high traffic loads. For example, for an ad hoc network with $\mathrm{N}=40$ stations, BUMA throughput is about $45 \%$ higher than that of DCF at full loading (Figure 5a).

The network throughput versus the number of stations of BUMA and DCF for ad hoc and infrastructure networks is shown in Figure 6 (a) and (b), respectively. It is found that BUMA has higher throughput than DCF irrespective of network architecture for $\mathrm{N}=10$ to 100 stations. For example, for an ad hoc network with $\mathrm{N}=20$ stations, BUMA's throughput is about $45 \%$ higher than that of DCF at $80 \%$ load (Figure 6a). The main conclusion that can be drawn from Figures 5 and 6 is that BUMA's throughput (both for individual stations and network wide) is significantly better than that ofDCF, especially under medium-to-high loads.

\section{Packet Delay}

The network mean packet delays of BUMA and DCF for $\mathrm{N}=40$ stations in ad hoc and infrastructure networks are shown in Figure 7 (a) and (b), respectively.

It is observed that BUMA achieves lower mean packet delay than DCF irrespective of network architecture, especially at load greater than $40 \%$. For example, for an ad hoc network with $\mathrm{N}=40$ stations, BUMA's mean packet delay is about $96 \%$ lower than DCF's at $70 \%$ load (Figure7a).

The network mean packet delays versus the number of active stations of BUMA and DCF for ad hoc and infrastructure networks are shown in Figure 8 (a) and (b), respectively. It is observed that BUMA's mean packet delays are lower than DCF's for both ad hoc and infrastructure networks. 
Figure 5. Network throughput against offered load of BUMA and DCF: (a) Ad hoc network; and (b) Infrastructure network

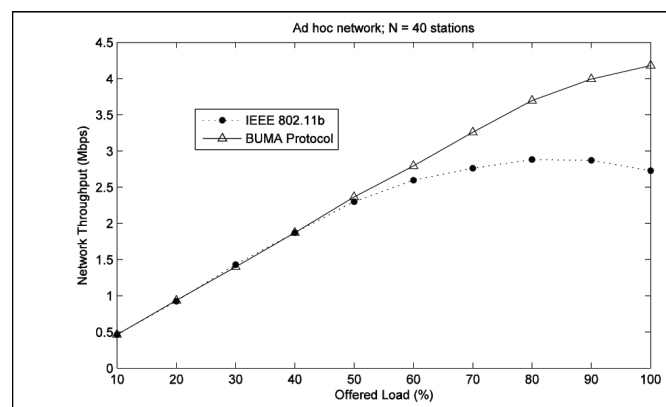

(a)

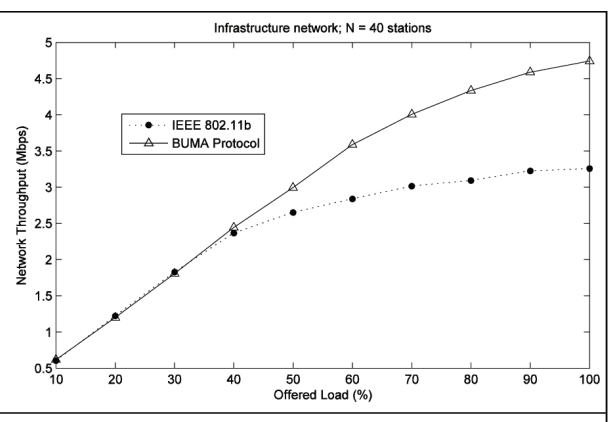

(b)

Figure 6. Network throughput versus number of active stations of BUMA and DCF: (a) Ad hoc network; (b) Infrastructure network

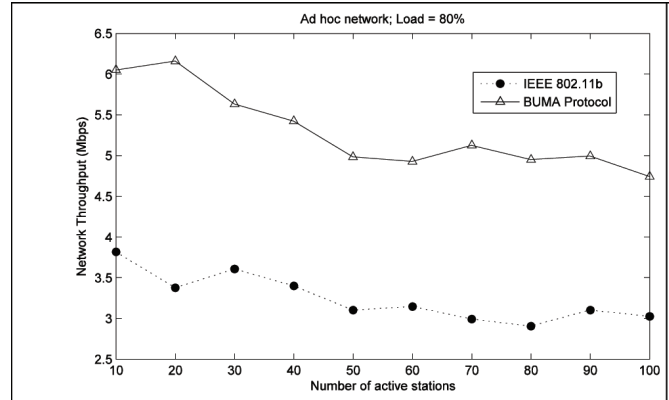

(a)

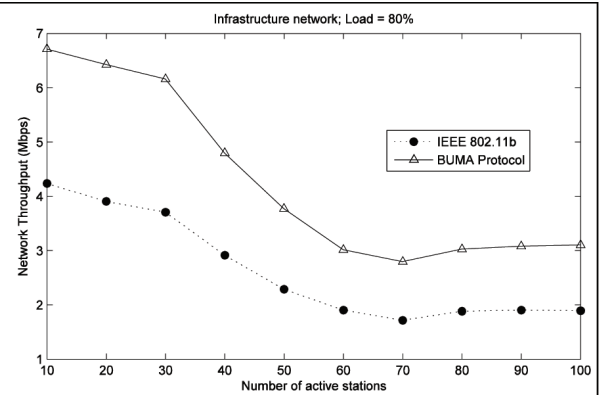

(b)

Figure 7. Mean packet delay versus offered load of BUMA and DCF: (a) Ad hoc network; and (b) Infrastructure network

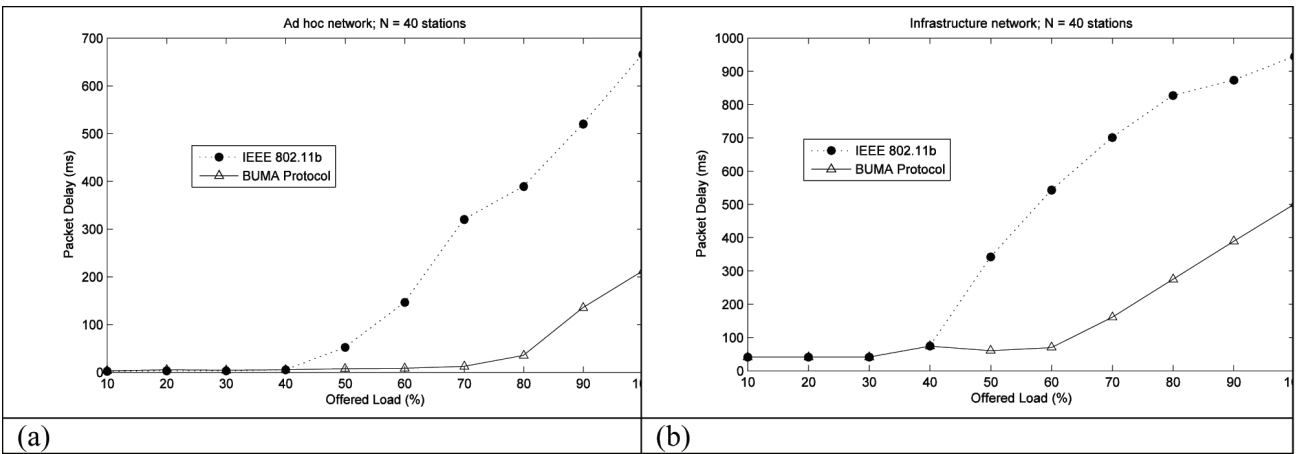


Figure 8. Mean packet delay versus number of active stations of BUMA and DCF: (a) Ad hoc network; and (b) Infrastructure network.

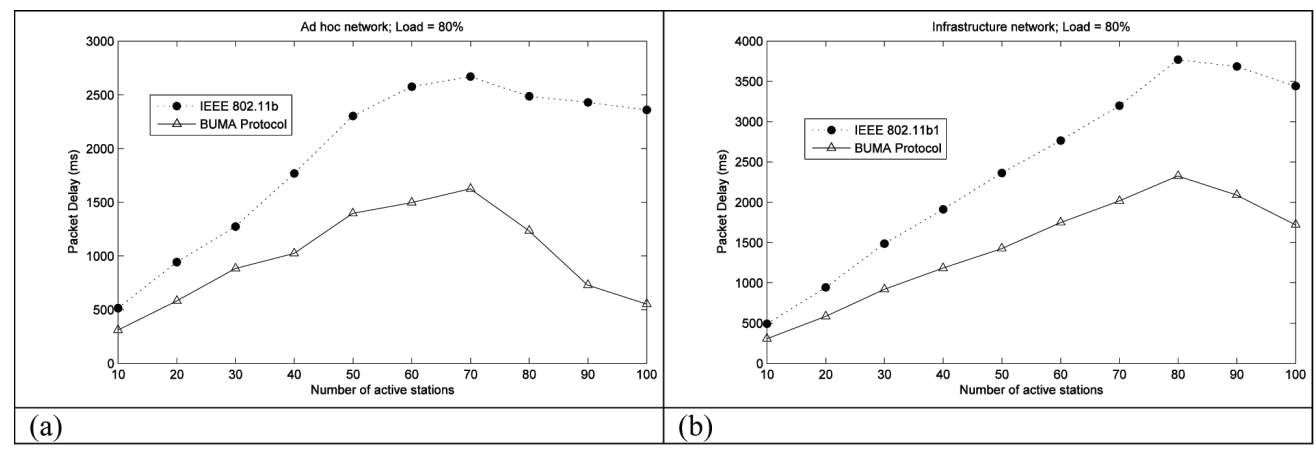

Figure 9. MDT fairness versus links of BUMA and DCF: (a) Ad hoc network; and (b) Infrastructure network.

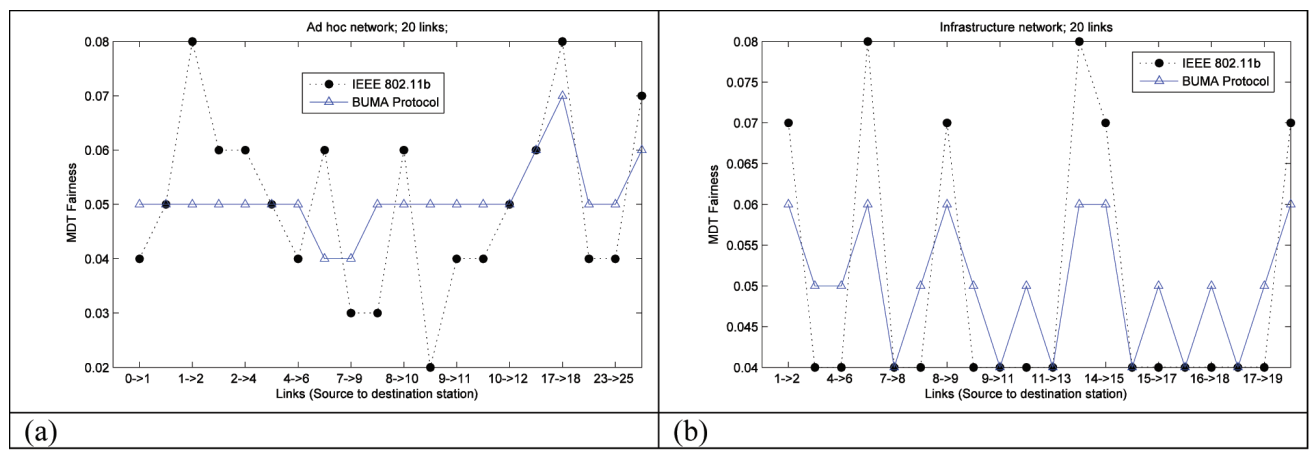

The main conclusion is that (Figures 7 and 8) stations using BUMA have a substantially lower mean packet delay than stations using DCF, especially under medium-to-high loads.

\section{Fairness Performance}

Figure 9 plots active links between source and destination stations (e.g. 0->1 indicates station 0 transmits a packet to station 1) versus MDT fairness for $\mathrm{N}=20$ stations for an ad hoc network (Figure 9a) and an infrastructure network (Figure 9b). It is observed that even though the proposed BUMA is not $100 \%$ fair in allocating bandwidth among active stations (for example, in an ideal case the throughput of each link should be about $5 \%$ of the total network throughput), it provides up to $50 \%$ higher MDT fairness than DCF (Figure 9a).

The main conclusion is that at $80 \%$ load MDT fairness (in both individual stations and network wide) of BUMA is significantly better than that of DCF.

The effects of offered load on the fairness of BUMA and DCF for $\mathrm{N}=10$ stations for ad hoc and infrastructure networks are shown in Figure 10 (a) and (b), respectively. The MDT fairness of BUMA is better than that of DCF irrespective of network architecture, especially under medium-to-high traffic loads (50 to $80 \%$ ). For example, for an infrastructure network at $50 \%$ load with $\mathrm{N}=10$ stations, BUMA has an 
Figure 10. MDT fairness versus offered load of BUMA and DCF: (a) Ad hoc network; and (b) Infrastructure network.

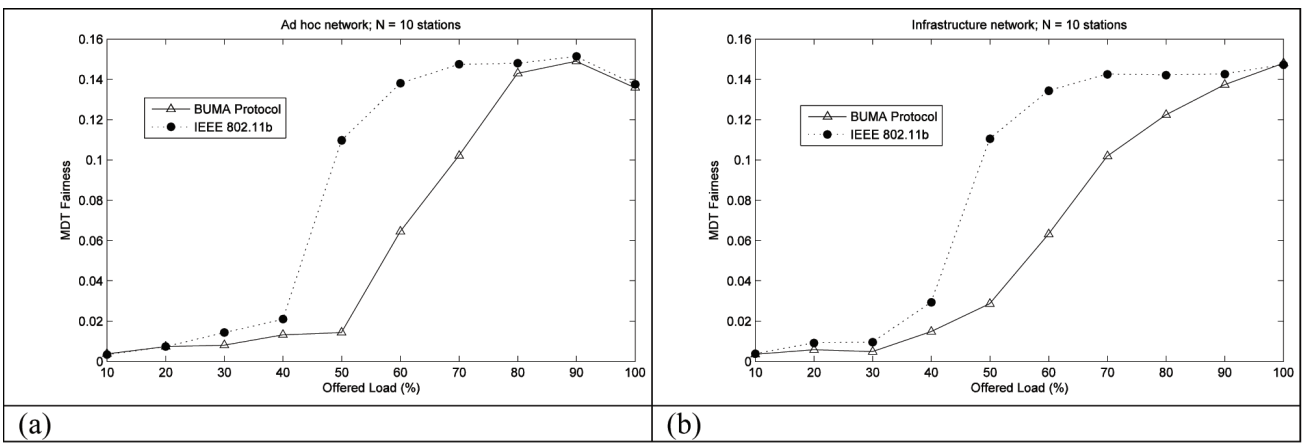

Figure 11. The effect of the number of active stations on MDT fairness of BUMA and DCF: (a) Ad hoc network; and (b) Infrastructure network.

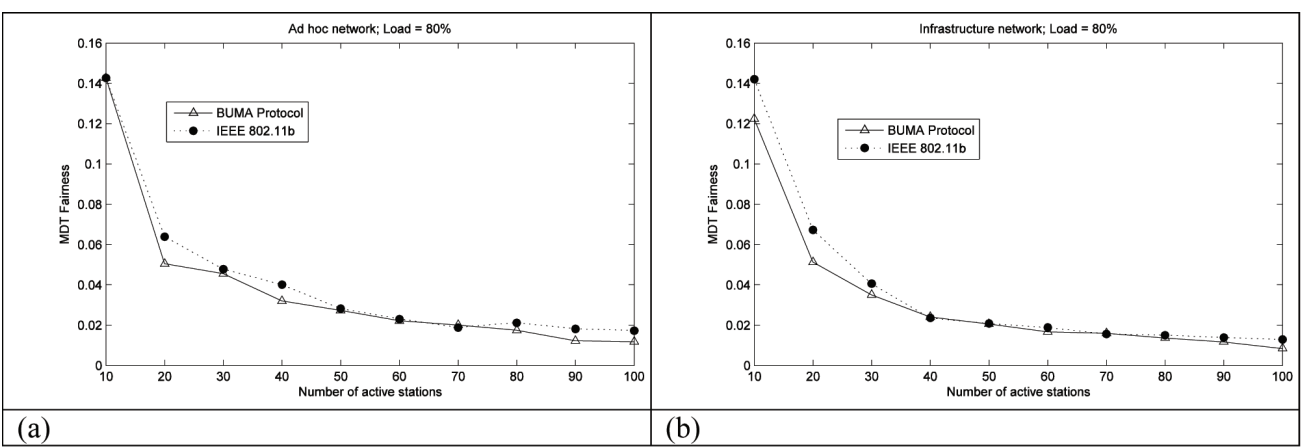

MDT about $8.2 \%$ lower than DCF's (Figure 10b). For offered load greater than $80 \%$, the fairness improvement is not very significant.

The effects of active stations on the MDT fairness of BUMA and DCF in ad hoc and infrastructure networks are demonstrated in Figure 11 (a) and (b), respectively. It is observed that BUMA has slightly better MDT fairness than that of DCF, especially for $\mathrm{N}>80$ stations at $80 \%$ load. However, this fairness improvement is not very significant.

The main conclusion (Figures 10 and 11) is that stations using BUMA achieve a slightly better MDT fairness than stations using DCF, especially under medium-to-high loads.

\section{Packet Drop Ratio}

The network-wide packet drop ratios of both the BUMA and 802.11b DCF protocols for $\mathrm{N}$ $=10$ to 100 stations at $80 \%$ offered load for an ad hoc and infrastructure network are shown in Figure 12 (a) and (b), respectively.

We observe that fewer packets are dropped under the BUMA protocol than the DCF protocol. For example, the BUMA offers about $28.5 \%$ lower packet drop ratio than the DCF for $\mathrm{N}=20$ stations at $80 \%$ offered load (Figure 12a). This improvement in packet dropping is due to the BUMA protocol's channel access strategy in which relatively fewer contentions 
Figure 12. Packet drop ratio versus the number of active stations of BUMA and DCF: (a) Ad hoc network; and (b) Infrastructure network.

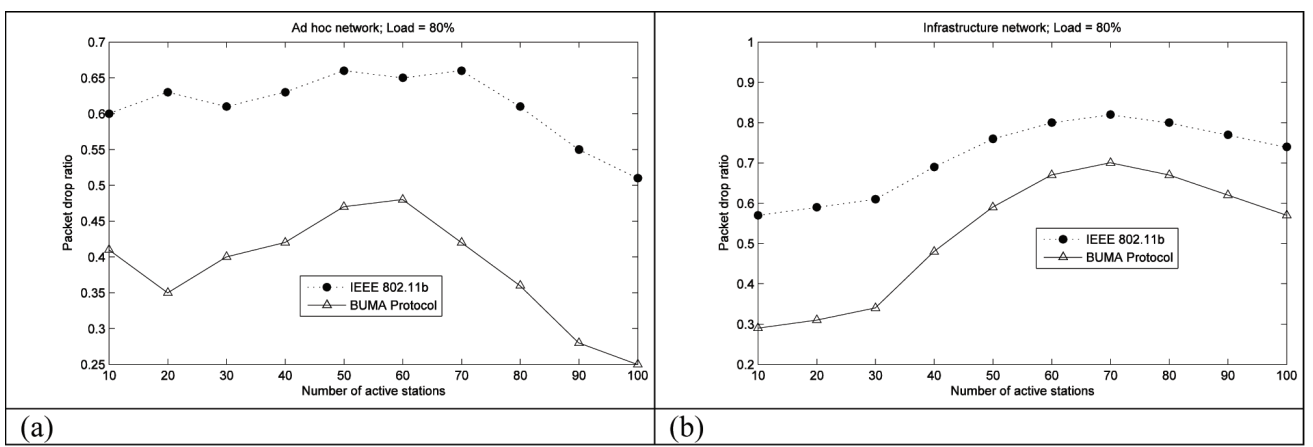

are faced by all active stations (i.e. fewer packet collisions) on the network, and consequently achieve high throughput performance than the DCF.

\section{IMPLICATIONS FOR SYSTEM IMPLIMENTATION}

The BUMA protocol is simple, easy to implement, and provides a low cost solution for improving WLAN performance. In the BUMA protocol, the temporary link layer buffer units at each station can easily be implemented using pre-existing random access memory (RAM) available in wireless devices, including laptops and APs. These buffer units can easily be created (for storing packets) and destroyed (after successful transmissions) at run time. Therefore, no additional hardware is required to implement the BUMA protocol.

With faster CPUs and RAM, the packetization delay at the stations will be minimal compared to the packet transmission time. The frame format of BUMA is designed in such a way that processing time for combining and decomposing frames is insignificant. The mechanisms for empty slot detection, slot synchronization, packet transmission, and packet reception can be implemented by firmware as is used in the 802.11 (IEEE Standards Association, 1997). When the destination station receives the BUMA frame, it decomposes the combined frame into normal frames, and acknowledges the last frame only. The destination station can easily identify boundaries of the combined frames using preambles and CRC.

In high traffic, BUMA performs better since the buffer unit can be filled up quickly with data from the upper protocol layers (e.g. IP datagram can be encapsulated up to the maximum length of the buffer unit) and hence carries larger payload with respect to protocol overheads. In light traffic BUMA performs as well as DCF by adapting buffer unit length to just one frame.

\section{CONCLUSION}

We improved the performance of a typical 802.11 WLAN by modifying the existing 802.11 DCF protocol which we called BUMA protocol. The BUMA achieved better system performance by including a temporary buffer unit at the MAC layer (for accumulating multiple packets for transmission). Simulation results have shown that the proposed BUMA achieved up to $45 \%$ higher throughput, $96 \%$ lower packet delay, $28.5 \%$ lower packet dropping, and 50\% greater fairness than the DCF for both ad hoc and infrastructure networks under medium-tohigh traffic loads. 
The BUMA does not change the operation of PHY layer and thus it can easily be implemented in the 802.11 networks requiring no hardware changes and no additional costs. BUMA can be used to improve the performance of all variants of 802.11 WLANs, including the $802.11 \mathrm{~b} / \mathrm{g}$. The models built using ns-2 simulator were validated using propagation measurements from wireless laptops and APs for an 802.11b WLAN. A good match between simulation results and measurements validated our simulation models.

The implementation aspect of BUMA has been discussed. RAM in wireless devices can be used to implement link layer buffer units at the stations without incurring any additional hardware costs. Fast modern processors and RAM render the station's packetization delay insignificant. A future paper will report on a rate adaptive QoS aware MAC protocol for multimedia WLANs.

\section{REFERENCES}

Aad, I., Ni, Q., Barakat, C., \& Turletti, T. (2005). Enhancing IEEE 802.11 MAC in congested environments. Computer Communications, 28(14), 1605-1617. doi:10.1016/j.comcom.2005.02.010

Banchs, A., Serrano, P., \& Azcorra, A. (2006). End-to-end delay analysis and admission control in 802.11 DCF WLANs. Communications of the ACM, 29(7), 842-854.

Banchs,A., \& Vollero, L. (2006). Throughputanalysis and optimal configuration of 802.11e EDCA. Computer Networks, 50(11), 1749-1768. doi:10.1016/j. comnet.2005.07.008

Bharghavan, V. (1994). MACAW: A media access protocol for wireless LANs. In Proceedings of the Annual Conference on Communications, Architectures, Protocols, and Applications (pp. 212-225). New York, NY: ACM Press.

Bianchi, G. (2000). Performance analysis of the IEEE 802.11 distributed coordination function. IEEE Journal on Selected Areas in Communications, 18(3), 535-547. doi:10.1109/49.840210
Bianchi, G., Borgonovo, F., Fratta, L., Musumeci, L., \& Zorzi, M. (1997). C-PRMA: A centralized packet reservation multiple access for local wireless communications. IEEE Transactions on Vehicular Technology, 46(2), 422-436. doi:10.1109/25.580781

Bianchi, G., Fratta, L., \& Oliveri, M. (1996, October 15-18). Performance evaluation and enhancement of the CSMA/CA MAC protocol for 802.11 wireless LANs. In Proceedings of the $7^{\text {th }}$ IEEE International Symposium on Personal, Indoor and Mobile Radio Communications, Taipei, Taiwan (pp. 392-396). Washington, DC: IEEE Computer Society.

Bruno, R., Conti, M., \& Gregori, E. (2002). Optimization of efficiency and energy consumption in p-persistent CSMA-based wireless LANs. IEEE Transactions on Mobile Computing, 1(1), 10-31. doi:10.1109/TMC.2002.1011056

Cali, F., \& Conti, M. (2000). Dynamic tuning of the IEEE 802.11 protocol to achieve a theoretical throughput limit. IEEE/ACM Transactions on Networking, 8(6), 785-799. doi:10.1109/90.893874

Chatzimisios, P., Vitsas, V., \& Boucouvalas, A. C. (2002). Throughput and delay analysis of IEEE 802.11 protocol. In Proceedings of the 5th IEEE International Workshop on Networked Appliances University, Liverpool, UK (pp. 168-174). Washington, DC: IEEE Computer Society.

Chieochan, S., Hossain, E., \& Diamond, J. (2010). Channel assignment schemes for infrastructure-based 802.11 WLANs: A survey. IEEE Communications Surveys \& Tutorials, 12(1), 124-136. doi:10.1109/ SURV.2010.020110.00047

Choudhury, S., \& Gibson, J. D. (2007). Payload length and rate adaptation for multimedia communications in wireless LANs. IEEE Journal on Selected Areas in Communications, 25(4), 796-807. doi:10.1109/ JSAC.2007.070515

David, M., Ken, D., \& Doug, L. (2007). Modeling the 802.11 distributed coordination function in nonsaturated heterogeneous conditions. IEEE/ ACM Transactions on Networking, 15(1), 159-172. doi:10.1109/TNET.2006.890136

Ergen, M., \& Varaiya, P. (2008). Formulation of distributed coordination function of IEEE 802.11 for asynchronous networks: Mixed data rate and packet size. IEEE Transactions on Vehicular Technology, 57(1), 436-447. doi:10.1109/TVT.2007.901887 
Ferre, P., Doufexi, A., Nix, A., \& Bull, D. (2004). Throughput analysis of IEEE 802.11 and IEEE 802.11 e MAC. In Proceedings of the IEEE Wireless Communications and Networking Conference, (Vol 2, pp. 783-788). Washington, DC: IEEE Computer Society.

Ganguly, S., Navda, V., Kim, K., Kashyap, A., Niculescu, D., \& Izmailov, R. (2006). Performance optimizations for deploying VoIP services in mesh networks. IEEE Journal on Selected Areas in Communications, 24(11), 2147-2158. doi:10.1109/ JSAC.2006.881594

IEEE Standards Association. (1997). IEEE 802.11 WG Standard for wireless LAN: Medium access control (MAC) and physical layer (PHY) specifications, IEEE 802.11 Standard. Retrieved from http://standards.ieee.org/getieee802/download/802.11-2007. pdf

IEEE Standards Association. (1999a). IEEE 802.11 Standard for Wireless LAN: Medium Access Control (MAC) and Physical Layer (PHY) Specification. Retrieved from http://standards.ieee.org/about/ get/802/802.11.html

IEEE Standards Association. (1999b). IEEE 802.11b WG, Part II: Wireless LAN medium access control (MAC) and physical layer (PHY) specifications: High-speed physical layer extension in the $2.4 \mathrm{GHz}$ band, IEEE 802.11b Standard. Retrieved from http:// standards.ieee.org/about/get/802/802.11.html

IEEE Standards Association. (2005). IEEE P802.11e Amendment to IEEE Std 802.11, Part II: Wireless LAN Medium Access Control (MAC) and Physical Layer (PHY) Specifications: MAC Quality of Server (QoS) Enhancements. Retrieved from http://standards.ieee. org/about/get/802/802.11.html

Jagadeesan, S., Manoj, B. S., \& Murthy, C. S. R. (2003). Interleaved carrier sense multiple access: An efficient MAC protocol for ad hoc wireless networks. In Proceedings of the IEEE International Conference on Communications, Anchorage, AK (Vol. 2, pp. 1124-1128). Washington, DC: IEEE Computer Society.

Jiang, L. B., \& Liew, S. C. (2008). Improving throughput and fairness by reducing exposed and hidden nodes in 802.11 networks. IEEE Transactions on Mobile Computing, 7(1), 34-49. doi:10.1109/ TMC.2007.1070

Kuo, W.-K. (2007). Energy efficiency modeling for IEEE 802.11 DCF system without retry limits. Computer Communications, 30(4), 856-862. doi:10.1016/j.comcom.2006.10.005
Kwon, Y., Fang, Y., \& Latchman, H. (2003). A novel $\mathrm{MAC}$ protocol with fast collision resolution for wireless LANs. In Proceedings of the $22^{\text {nd }}$ IEEE Computer and Communications Annual Joint Conference of INFOCOM (Vol. 2, pp. 853-862). Washington, DC: IEEE Computer Society.

Li, Y., \& Chen, I. (2010). Design and performance analysis of mobility management schemes based on pointer forwarding for wireless mesh networks. IEEE Transactions on Mobile Computing, 10(3), 349-361. doi:10.1109/TMC.2010.166

Lin, W.-Y., \& Wu, J.-S. (2007). Modified EDCF to improve the performance of IEEE 802.11e WLAN. Computer Communications, 30(4), 841-848. doi:10.1016/j.comcom.2006.10.013

Luo, J., Rosenberg, C., \& Girard,A. (2010). Engineering wireless mesh networks: Joint scheduling, routing, power control, and rate adaptation. IEEE/ACM Transactions on Networking, 18(5), 1387-1400. doi:10.1109/TNET.2010.2041788

Natkaniec, M., \& Pach, A. R. (2000, July 3-6). An analysis of backoff mechanism used in IEEE 802.11 networks. In Proceedings of the $5^{\text {th }}$ IEEE Symposium on Computers and Communications, Antibes-Juan les Pins, France (pp. 444-449). Washington, DC: IEEE Computer Society.

Natkaniec, M., \& Pach,A. R. (2002, October 27-30). PUMA - a new channel access protocol for wireless LANs. In Proceedings of the $5^{\text {th }}$ International Symposium on Wireless Personal Multimedia Communications (Vol. 3, pp. 1351-1355).

Ozugur, T., Naghshineh, M., Kermani, P., \& Copeland, J. A. (1999, December 5-9). Fair media access for wireless LANs. In Proceedings of the IEEE Global Telecommunications Conference, Rio de Janeireo, Brazil (Vol. 1, pp. 570-579). Los Alamitos, CA: IEEE Press.

Sarkar, N. I. (2006, January 2-4). Fairness studies of IEEE 802.11b DCF under heavy traffic conditions. In Proceedings of the First IEEE International Conference on Next-Generation Wireless Systems (pp. 11-16). Washington, DC: IEEE Computer Society.

Sarkar, N. I., \& Sowerby, K. W. (2006, November 27-30). Wi-Fi performance measurements in the crowded office environment: a case study. In Proceedings of the 10th IEEE International Conference on Communication Technology, Guilin, China (pp. 37-40). Washington, DC: IEEE Computer Society. 
Siringoringo, W., \& Sarkar, N. I. (2009). Teaching and learning Wi-Fi networking fundamentals using limited resources. In Gutierrez, J. (Ed.), Selected readings on telecommunications and networking (pp. 22-40). Hershey, PA: IGI Global.

Wang, W., Liew, S. C., \& Li, V. O. K. (2005). Solutions to performance problems in VoIP over a 802.11 wireless LAN. IEEE Transactions on Vehicular Technology, 54(1), 366-384. doi:10.1109/ TVT.2004.838890

Wang, Y., \& Garcia-Luna-Aceves, J. J. (2003, March 16-20). Throughput and fairness in a hybrid channel access scheme for ad hoc networks. In Proceedings of the IEEE Wireless Communications and Networking Conference (pp. 988-993). Washington, DC: IEEE Computer Society.

Xiao, Y. (2004, March 21-25). Concatenation and piggyback mechanisms for the IEEE 802.11 MAC. In Proceedings of the IEEE Wireless Communications and Networking Conference (pp. 1642-1647). Washington, DC: IEEE Computer Society.
Yin, J., Wang, X., \& Agrawal, D. P. (2005). Modeling and optimization of wireless local area network. Computer Communications, 28(10), 1204-1213. doi:10.1016/j.comcom.2004.07.027

You, T., Yeh, C.-H., \& Hassanein, H. (2003a). CSMA/ IC: A new class of collision-free MAC protocols for ad hoc wireless networks. In Proceedings of the Eighth IEEE International Symposium on Computers and Communication (pp. 843-848). Washington, DC: IEEE Computer Society.

You, T., Yeh, C.-H., \& Hassanein, H. (2003b, May 11-15). A new class of collision prevention MAC protocols for wireless ad hoc networks. In Proceedings of the IEEE International Conference on Communications, Anchorage, AK (pp. 1135-1140). Washington, DC: IEEE Computer Society.

Yun, L., Ke-Ping, L., Wei-Liang, Z., \& Qian-Bin, C. (2006). A novel random backoff algorithm to enhance the performance of IEEE 802.11 DCF. Wireless Personal Communications, 36(1), 29-44. doi:10.1007/s11277-006-6176-8 